\title{
Numerical solution and distinguishability in time fractional parabolic equation
}

\author{
Ali Demir ${ }^{1}$, Fatma Kanca ${ }^{2}$ and Ebru Ozbilge 3* $^{*}$
}

"Correspondence: ebru.ozbilge@ieu.edu.tr

${ }^{3}$ Department of Mathematics, Faculty of Science and Literature, Izmir University of Economics, Sakarya Caddesi, No.156, Balcova, Izmir 35330, Turkey Full list of author information is available at the end of the article

\begin{abstract}
This article deals with the mathematical analysis of the inverse problem of identifying the distinguishability of input-output mappings in the linear time fractional inhomogeneous parabolic equation $D_{t}^{\alpha} u(x, t)=\left(k(x) u_{x}\right)_{x}+r(t) F(x, t), 0<\alpha \leq 1$, with mixed boundary conditions $u(0, t)=\psi_{0}(t), u_{x}(1, t)=\psi_{1}(t)$. By defining the input-output mappings $\Phi[\cdot]: \mathcal{K} \rightarrow C^{1}[0, T]$ and $\Psi[\cdot]: \mathcal{K} \rightarrow C[0, T]$ the inverse problem is reduced to the problem of their invertibility. Hence, the main purpose of this study is to investigate the distinguishability of the input-output mappings $\Phi[\cdot]$ and $\Psi[\cdot]$. Moreover, the measured output data $f(t)$ and $h(t)$ can be determined analytically by a series representation, which implies that the input-output mappings $\Phi[\cdot]: \mathcal{K} \rightarrow C^{1}[0, T]$ and $\Psi[\cdot]: \mathcal{K} \rightarrow C[0, T]$ can be described explicitly, where $\Phi[r]=\left.k(x) u_{x}(x, t ; r)\right|_{x=0}$ and $\Psi[r]=\left.u(x, t ; r)\right|_{x=1}$. Also, numerical tests using finite difference scheme combined with an iterative method are presented.
\end{abstract}

\section{Introduction}

The inverse problem of unknown source function in a linear inhomogeneous parabolic equation by using over-measured data has generated an increasing amount of interest from engineers and scientist during the last few decades. This kind of problems play a crucial role in engineering, physics and applied mathematics. The problem of recovering an unknown source function in the mathematical model of a physical phenomenon is frequently encountered. Intensive study has been carried out on this kind of problem, and various inverse problems and many numerical methods developed. In papers [1-3] a coupled method for inverse source problem of spatial fractional anomalous diffusion equations and a boundary-type collocation method for inverse Cauchy inhomogeneous potential problems were considered. The inverse problem of an unknown coefficient in quasi-linear parabolic equations was studied by Demir and Ozbilge [4, 5]. Moreover, the existence and uniqueness of solutions for fractional differential equations with nonlocal and integral boundary conditions were studied by Ashyralyev and Sharifov [6]. Initial boundary-value problems for the one-dimensional time fractional diffusion equation were studied by Amanov and Ashyralyev in [7], the finite difference method for fractional parabolic equations with Neumann boundary conditions was studied by Ashyralyev and Cakir in [8], numerical solution of a fractional Schrodinger differential equation with the Dirichlet boundary condition was studied by Ashyralyev and Hicdurmaz in [9]. Moreover, Ashyralyev and Dal studied finite difference methods and iteration methods for fractional

(c) 2015 Demir et al. This article is distributed under the terms of the Creative Commons Attribution 4.0 International License (http://creativecommons.org/licenses/by/4.0/), which permits unrestricted use, distribution, and reproduction in any medium, provided you give appropriate credit to the original author(s) and the source, provide a link to the Creative Commons license, and indicate if changes were made. 
hyperbolic partial differential equations with the Neumann condition in [10]. Second order implicit finite difference schemes were applied to the right-hand side of the identification problem by Erdogan and Ashyralyev [11].

Fractional differential equations are generalizations of ordinary and partial differential equations to an arbitrary fractional order. By a linear time-fractional parabolic equation we mean certain parabolic-like partial differential equation governed by master equations containing fractional derivatives in time $[12,13]$. The research areas of fractional differential equations range from theoretical to applied aspects.

An inverse time independent source problem for a fractional diffusion equation is studied and analytical solution can be obtained based on the method of the eigenfunction expansion. Moreover, the uniqueness of the inverse problem is established by analytic continuation and Laplace transform. The efficiency and accuracy of the proposed computational method are supported by the numerical examples [14]. A time dependent inverse source problem with additional measurement data at an inner point for the fractional diffusion equation is investigated and stable and accurate numerical approximation is obtained by means of the boundary element method and the first order Tikhonov regularization. Results are verified by the numerical examples [15].

In this paper, the mathematical analysis of a time dependent inverse source problem with additional measurement data at a boundary point for the fractional diffusion equation is done. The distinguishability of the input-output mappings is investigated and the measured output data $f(t)$ and $h(t)$ can be constructed, which leads to the explicit form of the input-output mappings. It is shown that the distinguishability of the input-output mappings holds, which implies the injectivity of the inverse mappings $\Phi^{-1}$ and $\Psi^{-1}$.

Since fractional derivatives are necessarily nonlocal, the sensible models of nonlocal phenomena are made by means of them. In the modeling of the anomalous diffusion in porous media, the best approach was made by the use of the fractional derivatives since it includes the nonlocal phenomena and the anomalous behaviors can be governed by the derivative order. The analysis of the time-fractional porous medium equation was given in $[16,17]$, and a sensible model for the anomalous diffusion in porous medium equation was shown.

The main goal of this study is to investigate the distinguishability of the unknown source function via input-output mappings in a one-dimensional time fractional inhomogeneous parabolic equation. We first obtain the unique solution of this problem using the Fourier method of separation of variables with respect to the eigenfunctions of the corresponding Sturm-Liouville eigenvalue problem under certain conditions [18]. As the next step, the noisy free measured output data $f(t)$ and $h(t)$ are used to introduce the input-output mappings $\Phi[\cdot]: \mathcal{K} \rightarrow C^{1}[0, T]$ and $\Psi[\cdot]: \mathcal{K} \rightarrow C[0, T]$, where $\mathcal{K}$ represents the set of admissible source functions. The set of admissible source functions $\mathcal{K}$ includes all functions $r(t)$ such that problem (1) has a solution. Finally, we investigate the distinguishability of the unknown function $r(t)$ via the above input-output mappings $\Phi[\cdot]$ and $\Psi[\cdot]$.

Consider now the following initial boundary value problem:

$$
\left\{\begin{array}{l}
D_{t}^{\alpha} u(x, t)=\left(k(x) u_{x}\right)_{x}+r(t) F(x, t), \quad 0<\alpha \leq 1,(x, t) \in \Omega_{T}, \\
u(x, 0)=g(x), \quad 0<x<1, \\
u(0, t)=\psi_{0}(t), \quad u_{x}(1, t)=\psi_{1}(t), \quad 0<t<T,
\end{array}\right.
$$


where $\Omega_{T}=\left\{(x, t) \in R^{2}: 0<x<1,0<t \leq T\right\}$ and the fractional derivative $D_{t}^{\alpha} u(x, t)$ is defined in the Caputo sense $D_{t}^{\alpha} u(x, t)=\left(I^{1-\alpha} u^{\prime}\right)(t), 0<\alpha \leq 1, I^{\alpha}$ being the Riemann-Liouville fractional integral,

$$
\left(I^{\alpha} f\right)(t)= \begin{cases}\frac{1}{\Gamma(\alpha)} \int_{0}^{t}(t-\tau)^{\alpha-1} f(\tau) d \tau, & 0<\alpha \leq 1 \\ f(t), & \alpha=0 .\end{cases}
$$

The left and right boundary value functions $\psi_{0}(t)$ and $\psi_{1}(t)$ belong to $C[0, T]$. The functions $0<c_{0} \leq k(x)<c_{1}$ and $g(x)$ satisfy the following conditions:

(C1) $k(x) \in C^{1}[0,1]$,

(C2) $g(x) \in C^{2}[0,1], g(0)=\psi_{0}(0), g^{\prime}(1)=\psi_{1}(0)$.

Under these conditions, the initial boundary value problem (1) has the unique solution $u(x, t)$ defined in the domain $\bar{\Omega}_{T}=\left\{(x, t) \in R^{2}: 0 \leq x \leq 1,0 \leq t \leq T\right\}$ which belongs to the space $C\left(\bar{\Omega}_{T}\right) \cap W_{t}^{1}(0, T] \cap C_{x}^{2}(0,1)$. Moreover, it satisfies the equation, initial and boundary conditions. Note that the space $W_{t}^{1}(0, T]$ contains the functions $f \in C^{1}(0, T]$ such that $f^{\prime}(x) \in L(0, T)$.

Consider the inverse problem of determining the distinguishability of the unknown function $r(t)$ from the mixed type of measured output data at the boundaries $x=0$ and $x=1$, respectively,

$$
\begin{aligned}
& \Phi[r]=\left.k(x) u_{x}(x, t ; r)\right|_{x=0}, \quad r \in \mathcal{K} \subseteq C^{1}\left(\Omega_{T}\right), \\
& \Psi[r]=\left.u(x, t ; r)\right|_{x=1}, \quad r \in \mathcal{K} \subseteq C\left(\Omega_{T}\right) .
\end{aligned}
$$

Then, the inverse problem with the measured output data $f(t)$ and $h(t)$ can be formulated as follows:

$$
\begin{aligned}
& \Phi[r]=f, \quad f \in C^{1}(0, T], \\
& \Psi[r]=h, \quad h \in C(0, T] .
\end{aligned}
$$

These formulations reduce the inverse problem of determining an unknown function $r(t)$ to the problem of invertibility of the input-output mappings $\Phi[\cdot]$ and $\Psi[\cdot]$. This leads us to investigating the distinguishability of the source function via the above input-output mappings. We say that the mappings $\Phi[\cdot]: \mathcal{K} \rightarrow C^{1}[0, T]$ and $\Psi[\cdot]: \mathcal{K} \rightarrow C[0, T]$ have the distinguishability property if $\Phi\left[r_{1}\right] \neq \Phi\left[r_{2}\right]$ implies $r_{1}(t) \neq r_{2}(t)$ and $\Psi\left[r_{1}\right] \neq \Psi\left[r_{2}\right]$ implies $r_{1}(t) \neq r_{2}(t)$. This, in particular, means injectivity of the inverse mappings $\Phi^{-1}$ and $\Psi^{-1}$. In this paper, measured output data of Neumann type at the boundary $x=0$ and measured output data of Dirichlet type at the boundary $x=1$ are used in the determination of the distinguishability of the unknown function $r(t)$. In addition, in the distinguishability of the unknown function $r(t)$, analytical results are obtained.

The paper is organized as follows. In Section 2, analysis of the inverse problem with the single measured output data $f(t)$ at the boundary $x=0$ is given. Analysis of the inverse problem with the single measured output data $h(t)$ at the boundary $x=1$ is considered in Section 3. Numerical procedure and an example are given in Section 4. Finally, some concluding remarks are given in the last section. 


\section{Analysis of the inverse problem with given measured data $f(t)$}

Consider now the inverse problem with one measured output data $f(t)$ at $x=0$. In order to formulate the solution of parabolic problem (1) by using the Fourier method of separation of variables, let us first introduce an auxiliary function $v(x, t)$ as follows:

$$
v(x, t)=u(x, t)-\psi_{0}(t)-\psi_{1}(t) x, \quad x \in[0,1]
$$

by which we transform problem (1) into a problem with homogeneous boundary conditions. Hence the initial boundary value problem (1) can be rewritten in terms of $v(x, t)$ in the following form:

$$
\left\{\begin{array}{l}
D_{t}^{\alpha} v(x, t)-v_{x x}(x, t) \\
\quad=\left((k(x)-1) v_{x}(x, t)\right)_{x}-x D_{t}^{\alpha} \psi_{1}(t)-D_{t}^{\alpha} \psi_{0}(t)+k^{\prime}(x) \psi_{1}(t)+r(t) F(x, t), \\
v(x, 0)=g(x)-\psi_{0}(0)-\psi_{1}(0) x, \quad 0<x<1, \\
v(0, t)=0, \quad v_{x}(1, t)=0, \quad 0<t<T .
\end{array}\right.
$$

The unique solution of the initial-boundary value problem can be represented in the following form [18]:

$$
\begin{aligned}
v(x, t)= & \sum_{n=1}^{\infty}\left\langle\zeta(\theta), \phi_{n}(\theta)\right\rangle E_{\alpha, 1}\left(-\lambda_{n} t^{\alpha}\right) \phi_{n}(x) \\
& +\sum_{n=1}^{\infty}\left(\int _ { 0 } ^ { t } s ^ { \alpha - 1 } E _ { \alpha , \alpha } ( - \lambda _ { n } s ^ { \alpha } ) \left(\left\langle\xi(\theta, t-s), \phi_{n}(\theta)\right\rangle\right.\right. \\
& \left.\left.+\left\langle r(t-s) F(\theta, t-s), \phi_{n}(\theta)\right\rangle\right) d s\right) \phi_{n}(x),
\end{aligned}
$$

where

$$
\begin{aligned}
& \zeta(x)=g(x)-\psi_{0}(0)-\psi_{1}(0) x, \\
& \xi(x, t)=\left((k(x)-1) v_{x}(x, t)\right)_{x}-x D_{t}^{\alpha} \psi_{1}(t)-D_{t}^{\alpha} \psi_{0}(t)+k^{\prime}(x) \psi_{1}(t) .
\end{aligned}
$$

Moreover, $\left\langle\zeta(\theta), \phi_{n}(\theta)\right\rangle=\int_{0}^{1} \phi_{n}(\theta) \zeta(\theta) d \theta, E_{\alpha, \beta}$ being the generalized Mittag-Leffler function defined by

$$
E_{\alpha, \beta}(z)=\sum_{n=0}^{\infty} \frac{z^{n}}{\Gamma(\beta n+\alpha)} .
$$

Assume that $\phi_{n}(x)$ is the solution of the following Sturm-Liouville problem:

$$
\left\{\begin{array}{l}
-\phi_{x x}(x)=\lambda \phi(x), \quad 0<x<1, \\
\phi(0)=0, \quad \phi_{x}(1)=0, \quad 0<t<T .
\end{array}\right.
$$

The Neumann type of measured output data at the boundary $x=0$ can be written in terms of $v(x, t)$ in the following form:

$$
k(0)\left(v_{x}(0, t)+\psi_{1}(t)\right)=f(t), \quad t \in(0, T] .
$$


In order to arrange the above solution, let us define the following:

$$
\begin{aligned}
& z_{n}(t)=\left\langle\zeta(\theta), \phi_{n}(\theta)\right| E_{\alpha, 1}\left(-\lambda_{n} t^{\alpha}\right), \\
& w_{n}(t)=\int_{0}^{t} s^{\alpha-1} E_{\alpha, \alpha}\left(-\lambda_{n} s^{\alpha}\right)\left\langle\xi(\theta, t-s), \phi_{n}(\theta)\right\rangle d s, \\
& y_{n}(t)=\int_{0}^{t} s^{\alpha-1} E_{\alpha, \alpha}\left(-\lambda_{n} s^{\alpha}\right)\left\langle r(t-s) F(\theta, t-s), \phi_{n}(\theta)\right\rangle d s .
\end{aligned}
$$

The solution in terms of $z_{n}(t), w_{n}(t)$ and $y_{n}(t)$ can then be rewritten in the following form:

$$
v(x, t)=\sum_{n=1}^{\infty} z_{n}(t) \phi_{n}(x)+\sum_{n=1}^{\infty} w_{n}(t) \phi_{n}(x)+\sum_{n=1}^{\infty} y_{n}(t) \phi_{n}(x) .
$$

Differentiating both sides of the above identity with respect to $x$ and substituting $x=0$ yields

$$
v_{x}(0, t)=\sum_{n=1}^{\infty} z_{n}(t) \phi_{n}^{\prime}(0)+\sum_{n=1}^{\infty} w_{n}(t) \phi_{n}^{\prime}(0)+\sum_{n=1}^{\infty} y_{n}(t) \phi_{n}^{\prime}(0) .
$$

Taking into account the over-measured data $k(0)\left(v_{x}(0, t)+\psi_{1}(t)\right)=f(t)$,

$$
f(t)=k(0)\left(\psi_{1}(t)+\sum_{n=1}^{\infty} z_{n}(t) \phi_{n}^{\prime}(0)+\sum_{n=1}^{\infty} w_{n}(t) \phi_{n}^{\prime}(0)+\sum_{n=1}^{\infty} y_{n}(t) \phi_{n}^{\prime}(0)\right)
$$

is obtained, which implies that $f(t)$ can be determined analytically. The right-hand side of identity (5) defines the input-output mapping $\Phi[r]$ on the set of admissible source function $\mathcal{K}$ :

$$
\begin{aligned}
& \Phi[r](t):=k(0)\left(\psi_{1}(t)+\sum_{n=1}^{\infty} z_{n}(t) \phi_{n}^{\prime}(0)+\sum_{n=1}^{\infty} w_{n}(t) \phi_{n}^{\prime}(0)+\sum_{n=1}^{\infty} y_{n}(t) \phi_{n}^{\prime}(0)\right), \\
& \forall t \in[0, T] .
\end{aligned}
$$

The following lemma implies the relation between the source functions $r_{1}(t), r_{2}(t) \in \mathcal{K}$ at $x=0$ and the corresponding outputs $f_{j}(t):=k(0) u_{x}\left(0, t ; r_{j}\right), j=1,2$.

Lemma 1 Let $v_{1}(x, t)=v\left(x, t ; r_{1}\right)$ and $v_{2}(x, t)=v\left(x, t ; r_{2}\right)$ be the solutions of the direct problem (2), corresponding to the admissible parameters $r_{1}(t), r_{2}(t) \in \mathcal{K}$. If $f_{j}(t)=$ $k(0)\left(v_{x}\left(0, t ; r_{j}\right)+\psi_{1}(t)\right), j=1,2$, are the corresponding outputs, the outputs $f_{j}(t), j=1,2$, satisfy the following integral identity:

$$
\Delta f(t)=k(0)\left(\sum_{n=1}^{\infty} \Delta w_{n}(t) \phi_{n}^{\prime}(0)+\sum_{n=1}^{\infty} \Delta y_{n}(t) \phi_{n}^{\prime}(0)\right)
$$

for each $t \in(0, T]$, where $\Delta f(t)=f_{1}(t)-f_{2}(t), \Delta w_{n}(t)=w_{n}^{1}(t)-w_{n}^{2}(t), \Delta r(t)=r_{1}(t)-r_{2}(t)$ and $\Delta y_{n}(t)=y_{n}^{1}(t)-y_{n}^{2}(t)=\int_{0}^{t} s^{\alpha-1} E_{\alpha, \alpha}\left(-\lambda_{n} s^{\alpha}\right)\left\langle[\Delta r(t-s)] F(t-s), \phi_{n}(\theta)\right\rangle d s$. 
Proof By using identity (5), the measured output data $f_{j}(t):=k(0)\left(v_{x}(0, t)+\psi_{1}(t)\right), j=1,2$, can be written as follows:

$$
\begin{aligned}
& f_{1}(t)=k(0)\left(\psi_{1}(t)+\sum_{n=1}^{\infty} z_{n}^{1}(t) \phi_{n}^{\prime}(0)+\sum_{n=1}^{\infty} w_{n}^{1}(t) \phi_{n}^{\prime}(0)+\sum_{n=1}^{\infty} y_{n}^{1}(t) \phi_{n}^{\prime}(0)\right), \\
& f_{2}(t)=k(0)\left(\psi_{1}(t)+\sum_{n=1}^{\infty} z_{n}^{2}(t) \phi_{n}^{\prime}(0)+\sum_{n=1}^{\infty} w_{n}^{2}(t) \phi_{n}^{\prime}(0)+\sum_{n=1}^{\infty} y_{n}^{2}(t) \phi_{n}^{\prime}(0)\right)
\end{aligned}
$$

respectively. Hence the difference of these formulas implies the desired result.

The lemma and the definitions enable us to reach the following conclusion.

Corollary 1 Let the conditions of Lemma 1 hold. If in addition

$$
\left\langle r_{1}(t)-r_{2}(t), \phi_{n}(x)\right\rangle=0,
$$

$\forall t \in(0, T], \forall n=0,1, \ldots$ holds, then $f_{1}(t)=f_{2}(t), \forall t \in[0, T]$

Since $\phi_{n}(x), \forall n=0,1,2, \ldots$, form a basis for the space and $\phi_{n}^{\prime}(0) \neq 0, \forall n=0,1,2, \ldots$, then $r_{1}(t) \neq r_{2}(t)$ implies that $\left\langle r_{1}(t)-r_{2}(t), \phi_{n}(x)\right\rangle \neq 0$ at least for some $n \in \mathcal{N}$. Hence by Lemma 1 we conclude that $f_{1}(t) \neq f_{2}(t)$, which leads us to the following consequence: $\Phi\left[r_{1}\right] \neq \Phi\left[r_{2}\right]$ implies that $r_{1}(t) \neq r_{2}(t)$.

Theorem 1 Let conditions (C1), (C2) hold. Assume that $\Phi[\cdot]: \mathcal{K} \rightarrow C^{1}[0, T]$ is the input-output mapping defined by (6) and corresponding to the measured output $f(t):=$ $k(0) u_{x}(0, t)$. In this case the mapping $\Phi[r]$ has the distinguishability property in the class of admissible parameters $\mathcal{K}$, i.e.,

$$
\Phi\left[r_{1}\right] \neq \Phi\left[r_{2}\right] \quad \forall r_{1}, r_{2} \in \mathcal{K} \quad \Rightarrow \quad r_{1}(t) \neq r_{2}(t) .
$$

Proof From the above explanations the proof of the theorem is clear.

\section{Analysis of the inverse problem with given measured data $h(t)$}

Consider now the inverse problem with one measured output data $h(t)$ at $x=1$. Taking into account the over-measured data $h(t)=\left(v(1, t)+\psi_{0}(t)+\psi_{1}(t)\right)$,

$$
h(t)=\left(\psi_{0}(t)+\psi_{1}(t)+\sum_{n=1}^{\infty} z_{n}(t) \phi_{n}(1)+\sum_{n=1}^{\infty} w_{n}(t) \phi_{n}(1)+\sum_{n=1}^{\infty} y_{n}(t) \phi_{n}(1)\right)
$$

is obtained, which implies that $h(t)$ can be determined analytically. The right-hand side of identity (7) defines the input-output mapping $\Psi[r]$ on the set of admissible source functions $\mathcal{K}$ :

$$
\begin{aligned}
& \Psi[r](t):=\left(\psi_{0}(t)+\psi_{1}(t)+\sum_{n=1}^{\infty} z_{n}(t) \phi_{n}(1)+\sum_{n=1}^{\infty} w_{n}(t) \phi_{n}(1)+\sum_{n=1}^{\infty} y_{n}(t) \phi_{n}(1)\right), \\
& \forall t \in(0, T] .
\end{aligned}
$$


The following lemma implies the relation between the parameters $r_{1}(t), r_{2}(t) \in \mathcal{K}$ at $x=1$ and the corresponding outputs $h_{j}(t):=u\left(1, t ; r_{j}\right), j=1,2$.

Lemma 2 Let $v_{1}(x, t)=v\left(x, t ; r_{1}\right)$ and $v_{2}(x, t)=v\left(x, t ; r_{2}\right)$ be the solutions of the direct problem (2), corresponding to the admissible parameters $r_{1}(t), r_{2}(t) \in \mathcal{K}$. If $h_{j}(t)=v\left(1, t ; r_{j}\right)+$ $\psi_{1}(t)+\psi_{0}(t), j=1,2$, are the corresponding outputs, the outputs $h_{j}(t), j=1,2$, satisfy the following integral identity:

$$
\Delta h(t)=\sum_{n=1}^{\infty} \Delta w_{n}(t) \phi_{n}(1)+\sum_{n=1}^{\infty} \Delta y_{n}(t) \phi_{n}(1)
$$

for each $t \in(0, T]$, where $\Delta h(t)=h_{1}(t)-h_{2}(t), \Delta w_{n}(t)=w_{n}^{1}(t)-w_{n}^{2}(t), \Delta r(t)=r_{1}(t)-r_{2}(t)$.

Proof By using identity (7), the measured output data $h_{j}(t):=v(1, t)+\psi_{0}(t)+\psi_{1}(t), j=1,2$, can be written as follows:

$$
\begin{aligned}
& h_{1}(t)=\psi_{0}(t)+\psi_{1}(t)+\sum_{n=1}^{\infty} z_{n}^{1}(t) \phi_{n}(1)+\sum_{n=1}^{\infty} w_{n}^{1}(t) \phi_{n}(1)+\sum_{n=1}^{\infty} y_{n}^{1}(t) \phi_{n}(1), \\
& h_{2}(t)=\psi_{0}(t)+\psi_{1}(t)+\sum_{n=1}^{\infty} z_{n}^{2}(t) \phi_{n}(1)+\sum_{n=1}^{\infty} w_{n}^{2}(t) \phi_{n}(1)+\sum_{n=1}^{\infty} y_{n}^{2}(t) \phi_{n}(1),
\end{aligned}
$$

respectively. Since $z_{n}^{1}(t)=z_{n}^{2}(t)$ from the definition then the difference of these formulas implies the desired result.

Corollary 2 Let the conditions of Lemma 2 hold. If in addition

$$
\left\langle r_{1}(t)-r_{2}(t), \phi_{n}(x)\right\rangle=0, \quad \forall n=0,1, \ldots
$$

holds, then $h_{1}(t)=h_{2}(t), \forall t \in(0, T]$.

Since $\phi_{n}(x), \forall n=0,1,2, \ldots$, form a basis for the space and $\phi_{n}^{\prime}(0) \neq 0, \forall n=0,1,2, \ldots$, then $r_{1}(t) \neq r_{2}(t)$ implies that $\left\langle r_{1}(t)-r_{2}(t), \phi_{n}(x)\right\rangle \neq 0$ at least for some $n \in \mathcal{N}$. Hence by Lemma 2 we conclude that $h_{1}(t) \neq h_{2}(t)$, which leads us to the following consequence: $\Psi\left[r_{1}\right] \neq \Psi\left[r_{2}\right]$ implies that $r_{1}(t) \neq r_{2}(t)$.

Theorem 2 Let conditions (C1), (C2) hold. Assume that $\Psi[\cdot]: \mathcal{K} \rightarrow C[0, T]$ is the inputoutput mapping defined by (8) and corresponding to the measured output $h(t):=u(1, t)$. In this case the mapping $\Psi[r]$ has the distinguishability property in the class of admissible parameters $\mathcal{K}$, i.e.,

$$
\Psi\left[r_{1}\right] \neq \Psi\left[r_{2}\right] \quad \forall r_{1}, r_{2} \in \mathcal{K} \quad \Rightarrow \quad r_{1}(t) \neq r_{2}(t)
$$

Proof From the above explanations the proof of the theorem is clear. 


\section{Numerical procedure}

We use finite difference method to problem (1). We subdivide the intervals $[0,1]$ and $[0, T]$ into $M$ and $N$ subintervals of equal lengths $h=\frac{1}{M}$ and $\tau=\frac{T}{N}$, respectively. The first order implicit scheme of problem (1) is as follows [19]:

$$
\begin{aligned}
& \quad \frac{1}{\Gamma(1-\alpha)} \sum_{k=1}^{j} \frac{\Gamma(j-k-\alpha+1)}{(j-k) !}\left(\frac{u_{i}^{k}-u_{i}^{k-1}}{\tau^{\alpha}}\right) \\
& \quad=\frac{1}{h}\left(k_{i+1} \frac{u_{i+1}^{j}-u_{i}^{j}}{h}-k_{i} \frac{u_{i}^{j}-u_{i-1}^{j}}{h}\right)+r^{j} F_{i}^{j}, \\
& u_{i}^{0}=g_{i}, \\
& u_{0}^{j}=\psi_{0}^{j}, \\
& u_{M+1}^{j}=\left(u_{M}^{j}+\psi_{1}^{j}\right) h,
\end{aligned}
$$

where $1 \leq i \leq M$ and $0 \leq j \leq N$ are the indices for the spatial and time steps, respectively, $u_{i}^{j}=u\left(x_{i}, t_{j}\right), r^{j}=r\left(t_{j}\right), g_{i}=g\left(x_{i}\right), F_{i}^{j}=F\left(x_{i}, t_{j}\right), \psi_{0}^{j}=\psi_{0}\left(t_{j}\right), \psi_{1}^{j}=\psi_{1}\left(t_{j}\right), x_{i}=i h, t_{j}=j \tau$. At the $t=0$ level, adjustment should be made according to the initial condition and the compatibility requirements.

Now, let us construct the predicting-correcting mechanism. Firstly, if we use the measured output data is $u(1, t)=h(t)$, we obtain

$$
r(t)=\frac{D_{t}^{\alpha} h(t)-\left(k(1) u_{x}(1, t)\right)_{x}}{F(1, t)} .
$$

The finite difference approximation of $r(t)$ is

$$
r^{j}=\frac{\left[H^{j}-\frac{1}{h}\left(k_{M+1} \psi 1^{j}-k_{M} \frac{u_{M}^{j}-u_{M-1}^{j}}{h}\right)\right]}{F_{M}^{j}},
$$

where $H^{j}=D_{t}^{\alpha} h\left(t_{j}\right), j=0,1, \ldots, N$.

In numerical computation, since the time step is very small, we can take $r^{j(0)}=r^{j-1}$, $u_{i}^{j(0)}=u_{i}^{j-1}, j=0,1,2, \ldots, N, i=1,2, \ldots, M$. At each $s$ th iteration step we first determine $r^{j(s)}$ from the formula

$$
r^{j(s)}=\frac{\left[H^{j}-\frac{1}{h}\left(k_{M+1} \psi_{1}^{j}-k_{M} \frac{u_{M}^{j(s)}-u_{M-1}^{j(s)}}{h}\right)\right]}{F_{M}^{j}} .
$$

Then from (9)-(12) we obtain

$$
\begin{aligned}
& \frac{1}{\Gamma(1-\alpha)} \sum_{k=1}^{j} \frac{\Gamma(j-k-\alpha+1)}{(j-k) !}\left(\frac{u_{i}^{k(s)}-u_{i}^{k(s-1)}}{\tau^{\alpha}}\right) \\
& \quad=\frac{1}{h}\left(k_{i+1} \frac{u_{i+1}^{j(s)}-u_{i}^{j(s)}}{h}-k_{i} \frac{u_{i}^{j(s)}-u_{i-1}^{j(s)}}{h}\right)+r^{j(s)} F_{i}^{j}, \\
& u_{i}^{0}=g_{i},
\end{aligned}
$$




$$
\begin{aligned}
& u_{0}^{j(s)}=\psi_{0}^{j}, \\
& u_{M}^{j(s)}=\left(u_{M-1}^{j}+\psi 1^{j}\right) h, \quad s=0,1,2, \ldots .
\end{aligned}
$$

The system of equations (14)-(17) can be solved by the Gauss elimination method and $u_{i}^{j(s)}$ is determined. If the difference of values between two iterations reaches the prescribed tolerance, the iteration is stopped and we accept the corresponding values $r^{j(s)}$, $u_{i}^{j(s)}\left(i=1,2, \ldots, N_{x}\right)$ as $r^{j}, u_{i}^{j}\left(i=1,2, \ldots, N_{x}\right)$, on the $(j)$ th time step, respectively. In virtue of this iteration, we can move from level $j$ to level $j+1$.

Example 1 Consider the following problem for $\alpha=1 / 2$ :

$$
\begin{aligned}
& D_{t}^{1 / 2} u(x, t)=\left(e^{x} u_{x}\right)_{x}+r(t)\left[\left(\frac{16}{5 \sqrt{\pi}} \sqrt{t}+\frac{\pi^{2}}{4} t e^{x}\right) \sin \frac{\pi}{2} x-\frac{\pi}{2} t e^{x} \cos \frac{\pi}{2} x\right], \\
& u(x, 0)=0, \\
& u(0, t)=0, \\
& u_{x}(1, t)=0,
\end{aligned}
$$

and the measured output data is $h(t)=t^{3}$.

The exact solution of this problem is $\{r(t), u(x, t)\}=\left\{t^{2}, t^{3} \sin \frac{\pi}{2} x\right\}$.

Let us apply the scheme above for the step sizes $h=0.05, \tau=0.05$. Figures 1,2 show the exact and the numerical solutions of $\{r(t), u(x, t)\}$ when $T=1 / 2$.

From these figures it can be seen that the agreement between the numerical and exact solutions for $r(t)$ and $u(x, T)$ is excellent.

Next, we will illustrate the stability of the numerical solution with respect to the noisy overdetermination data, defined by the function

$$
h_{\gamma}(t)=h(t)(1+\gamma \theta)
$$

where $\gamma$ is the percentage of noise and $\theta$ are random variables generated from a uniform distribution in the interval $[-1,1]$.

In the case when $T=1 / 2$, the illustrations of the sensitivity of the scheme with respect to noisy overdetermination data are shown in Figures 3, 4 and 5.

Figure 1 The exact and numerical solutions of $r(t)$. The exact solution is shown with a dashed line.

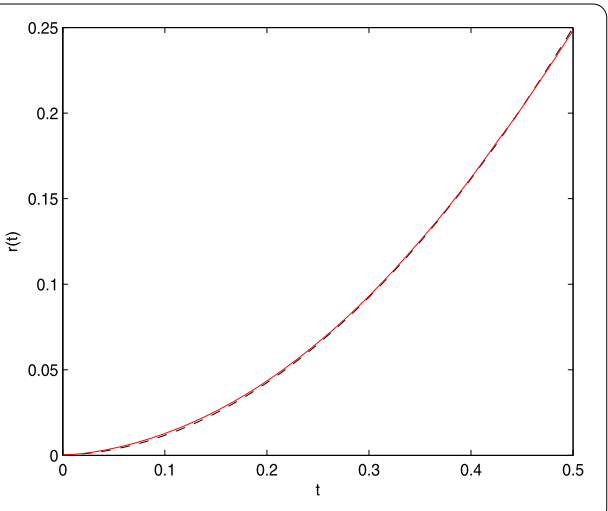


Figure 2 The exact and numerical solutions of $\boldsymbol{u}(\boldsymbol{x}, \boldsymbol{T})$. The exact solution is shown with a dashed line.

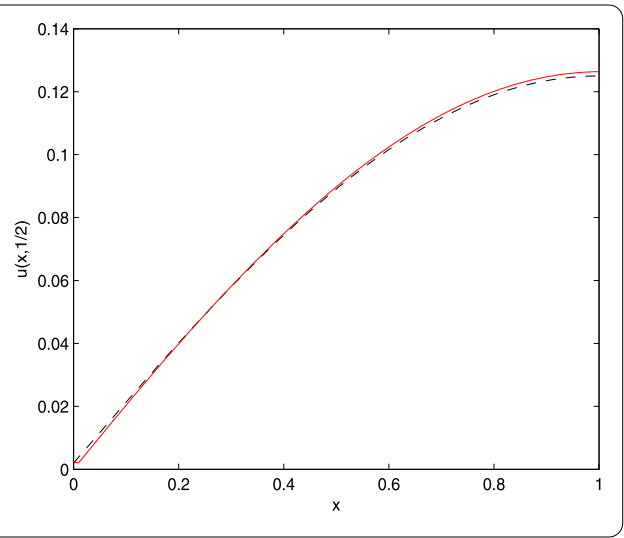

Figure 3 The exact and numerical solutions of $r(t)$ for $1 \%$ noisy data. The exact solution is shown with a dashed line.

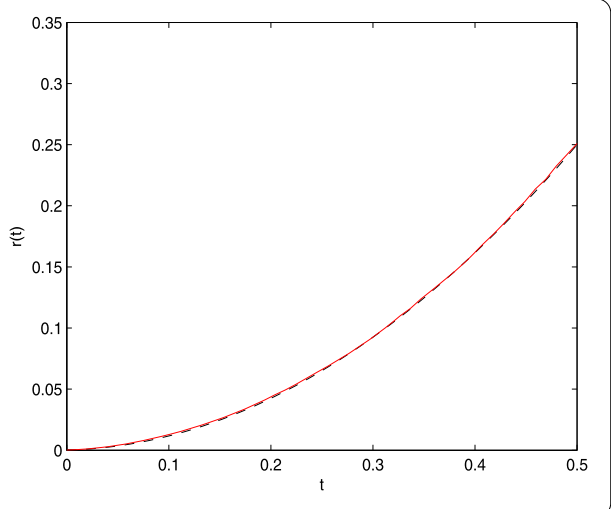

Figure 4 The exact and numerical solutions of $r(t)$ for $3 \%$ noisy data. The exact solution is shown with a dashed line.

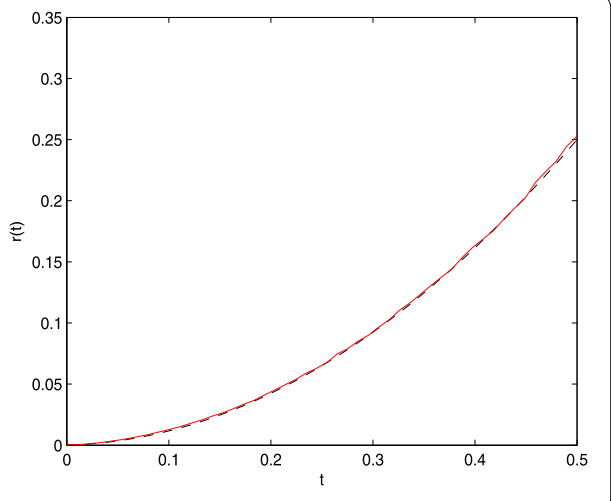

Example 2 In the previous Example 1, a smooth function given by $r(t)=t^{2}$ is considered. In Example 2, a more severe discontinuous test function is given:

$$
r(t)= \begin{cases}-t^{2}, & t \in\left[0, \frac{1}{4}\right), \\ t^{2}, & t \in\left[\frac{1}{4}, \frac{1}{2}\right] .\end{cases}
$$

Let us apply the scheme above for the step sizes $h=0.05, \tau=0.05$. Figure 6 shows the exact and the numerical solutions of $r(t)$ when $T=1 / 2$. 
Figure 5 The exact and numerical solutions of $r(t)$ for $\mathbf{5 \%}$ noisy data. The exact solution is shown with a dashed line.

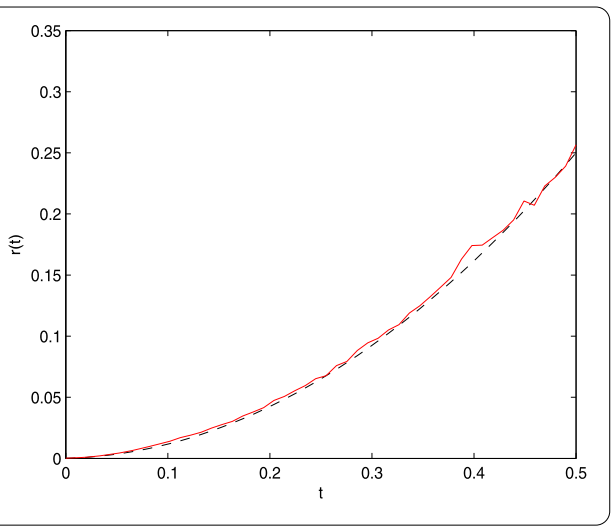

Figure 6 The exact and numerical solutions of $r(t)$. The exact solution is shown with a dashed line.

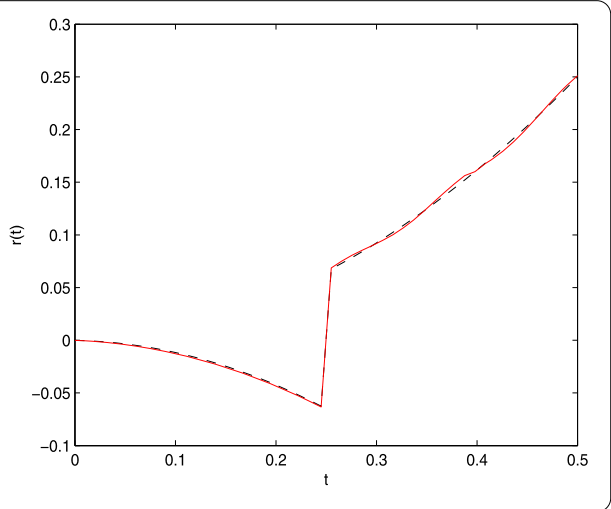

\section{Some discussions}

In the previous section, in Example 1, the man-made noise in the measured output data is added to show the stability of the numerical method. From Figures 3, 4 and 5 it can be seen that the results are quite stable for small noise in the input data. Also in Example 2, a discontinuous source function is given to show the efficiency of the present method. From Figure 6 it can be seen that the agreement between the numerical and exact solutions for $r(t)$ is excellent.

\section{Conclusion}

The aim of this study was to investigate the distinguishability properties of the inputoutput mappings $\Phi[\cdot]: \mathcal{K} \rightarrow C[0, T]$ and $\Psi[\cdot]: \mathcal{K} \rightarrow C^{1}[0, T]$, which are determined by the measured output data at $x=0$ and $x=1$, respectively. In this study, we conclude that the distinguishability of the input-output mappings holds, which implies the injectivity of the inverse mappings $\Phi^{-1}$ and $\Psi^{-1}$. The measured output data $f(t)$ and $h(t)$ are obtained analytically by a series representation, which leads to the explicit form of the input-output mappings $\Phi[\cdot]$ and $\Psi[\cdot]$. This work advances our understanding of the use of the Fourier method of separation of variables and the input-output mapping in the investigation of inverse problems for fractional parabolic equations. The author plans to consider various fractional inverse problems in future studies since the method discussed has a wide range of applications. 
Authors' contributions

All authors contributed equally to the writing of this paper. All authors read and approved the final manuscript.

\section{Author details}

'Department of Mathematics, Kocaeli University, Umuttepe, Izmit, Kocaeli 41380, Turkey. ${ }^{2}$ Department of Management Information Systems, Kadir Has University, Istanbul, 34083, Turkey. ${ }^{3}$ Department of Mathematics, Faculty of Science and Literature, Izmir University of Economics, Sakarya Caddesi, No.156, Balcova, Izmir 35330, Turkey.

\section{Acknowledgements}

The research was supported in parts by the Scientific and Technical Research Council (TUBITAK) of Turkey, Izmir University of Economics, Kocaeli University and Kadir Has University.

Received: 23 March 2015 Accepted: 28 July 2015 Published online: 16 August 2015

\section{References}

1. Wei, H, Chen, W, Sun, H, Li, X: A coupled method for inverse source problem of spatial fractional anomalous diffusion equations. Inverse Probl. Sci. Eng. 18(7), 945-956 (2010)

2. Chen, W, Fu, ZJ: Boundary particle method for inverse Cauchy problems of inhomogeneous Helmholtz equations J. Mar. Sci. Technol. 17(3), 157-163 (2009)

3. Fu, ZJ, Chen, W, Zhang, CZ: Boundary particle method for Cauchy inhomogeneous potential problems. Inverse Probl. Sci. Eng. 20(2), 189-207 (2012)

4. Demir, A, Ozbilge, E: Analysis of a semigroup approach in the inverse problem of identifying an unknown coefficient. Math. Methods Appl. Sci. 31, 1635-1645 (2008)

5. Demir, A, Ozbilge, E: Semigroup approach for identification of the unknown coefficient in a quasi-linear parabolic equation. Math. Methods Appl. Sci. 30, 1283-1294 (2007)

6. Ashyralyev, A, Sharifov, Y: Existence and uniqueness of solutions for the system of nonlinear fractional differential equations with nonlocal and integral boundary conditions. Abstr. Appl. Anal. 2012, 594802 (2012)

7. Amanov, D, Ashyralyev, A: Initial-boundary value problem for fractional partial differential equations of higher order. Abstr. Appl. Anal. 2012, 9733102 (2012)

8. Ashyralyev, A, Cakir, Z: FDM for fractional parabolic equations with the Neumann condition. Adv. Difference Equ. 2013, $120(2013)$

9. Ashyralyev, A, Hicdurmaz, B: On the numerical solution of fractional Schrodinger differential equations with the Dirichlet condition. Int. J. Comput. Math. 89, 1927-1936 (2012)

10. Ashyralyev, A, Dal, F: Finite difference and iteration methods for fractional hyperbolic partial differential equations with the Neumann condition. Discrete Dyn. Nat. Soc. 2012, 434976 (2012)

11. Erdogan, AS, Ashyralyev, A: On the second order implicit difference schemes for a right hand side identification problem. Appl. Math. Comput. 226, 212-229 (2014)

12. Renardy, M, Rogers, RC: An Introduction to Partial Differential Equations. Springer, New York (2004)

13. Showalter, RE: Monotone Operators in Banach Spaces and Nonlinear Partial Differential Equations. Am. Math. Soc., Providence (1997)

14. Zhang, Y, Xiang, $X$ : Inverse source problem for a fractional diffusion equation. Inverse Probl. 27(3), 035010 (2011)

15. Wei, T, Zhang, ZQ: Reconstruction of a time-dependent source term in a time-fractional diffusion equation. Eng. Anal. Bound. Elem. 37(1), 23-31 (2013)

16. Plociniczak, L: Approximation of the Erdelyi-Kober operator with application to the time-fractional porous medium equation. SIAM J. Appl. Math. 74(4), 1219-1237 (2014)

17. Plociniczak, L: Analytical studies of a time-fractional porous medium equation. Derivation, approximation and applications. Commun. Nonlinear Sci. Numer. Simul. 24(1), 169-183 (2015)

18. Luchko, Y: Initial boundary value problems for the one dimensional time-fractional diffusion equation. Fract. Calc. Appl. Anal. 15, 141-160 (2012)

19. Ashyralyev, A: A note on fractional derivatives and fractional powers of operators. J. Math. Anal. Appl. 357, 232-236 (2009)

\section{Submit your manuscript to a SpringerOpen ${ }^{\circ}$ journal and benefit from:}

- Convenient online submission

- Rigorous peer review

- Immediate publication on acceptance

Open access: articles freely available online

High visibility within the field

- Retaining the copyright to your article 